\title{
Investigation on the Dynamic Behaviour of an Ultrasonic-Levitation Magnetic Guiding System
}

\author{
Jan Reiners $^{1 \mathrm{a}}$ and Berend Denkena ${ }^{1 \mathrm{~b}}$ \\ ${ }^{1}$ Institute of Production Engineering and Machine Tools (IFW), Leibniz Universität Hannover, Germany \\ areiners@ifw.uni-hannover.de, ${ }^{\mathrm{b}}$ denkena@ifw.uni-hannover.de
}

Keywords: Micro machining, guiding system, ultrasonic levitation

\begin{abstract}
Guiding systems for precision manufacturing machines have to fulfil high demands. Low compliance and the absence of friction is required to achieve a high production accuracy. This article presents a novel active guiding system based on the combination of ultrasonic levitation and magnetic actuators. Firstly, the combined actuator and its working principles are described. Subsequently, the concept for an active, frictionless and medium-free guiding system is outlined. In addition to the free degree of freedom (DOF) in guiding direction, the other 5 DOF are adjustable in order to improve the guides positioning accuracy. The experimental validation of the concept is conducted with a simplified prototype, acting as a 3 DOF adjustable planar guide. Finally, measured compliance frequency responses demonstrate the performance of this novel active guiding concept.
\end{abstract}

\section{Introduction}

A multitude of daily goods e.g. smartphones, TVs etc. contain components, that require a production accuracy in a range of micrometres or less. Nowadays even optical surfaces are manufactured by multi-axis precision machine tools [1]. Their guiding systems influence the manufacturing accuracy substantially. Contactless guiding systems, such as hydro- and aerostatic systems, are common in machine tools for precision manufacturing due to low friction and wear [2]. Hydrostatic guiding systems are characterized by a low friction coefficient of $\mu=[0.001 \ldots 0.006]$ and a high damping. Disadvantageous are the technical effort to pressurize and recirculate the oil. Aerostatic guiding systems use air as guiding fluid, instead of oil. On the one hand, this leads to an even lower friction coefficient down to $\mu=\left[10^{-7} \ldots 10^{-6}\right]$ [3]. On the other hand, induced by the low viscosity of air, air gaps $<10 \mu \mathrm{m}$ are necessary to achieve sufficient compliance [4]. Thus, even minor form- and parallelism deviations of the guiding rail directly reduce the positioning accuracy of the guiding system significantly. In addition to that, thermal growth results in further deviations. Hence, close manufacturing tolerances, expenditures for tempering as well as fluid preparation and pressurization increase the cost of these guiding systems.

In order to improve the applicability of guiding systems for precision manufacturing, scholars have researched active guiding systems as well. Electromagnetically preloaded aerostatic guiding systems eliminate the load dependence of the air cushion and improve the system's compliance substantially [5]. Guiding systems exclusively based on controlled electromagnets supersede most of the mentioned drawbacks [6]. Especially their inherent fine positioning capabilities can compensate inaccuracies of the guiding system (e.g. manufacturing errors, thermal growth). Depending on the applied control system, additional active damping capabilities are realisable in order to adapt the machine tools structural dynamic during the manufacturing process [7]. Current research transfers these capabilities to ultra-precision manufacturing systems [8]. However, the effort for the implementation of the control system are extensive. Additionally, a necessary mechanical wrap around results in large and complex magnetic guided structures.

The combination of ultrasonic levitation actuators with magnetic actuators offers the potential to develop a contactless, medium free and active adaptable guiding system, which supersedes a mechanical wrap around. By the use of ultrasonic levitation, an aerostatic-similar but controllable air cushion is induced. The cushion is generated from the ambient air, without the need of a medium feeding. An additional controllable magnetic actuator adds a preload. Hence, the force generation of 
the combined actuator operates bidirectional, a mechanical wrap around is not required. By controlling the forces of both technologies, the combined ultrasonic-levitation magnetic actuator operates as an integrated fine positioning system as well as an active damping control.

\section{Combined ultrasonic levitation magnetic actuator}

This novel guiding concept uses the combined ultrasonic-levitation-magnetic (USLM) actuator concept [9] which is described by [10] in detail. Fig. 1 (a) shows the combined actuator, mounted on the slide of a guiding system.

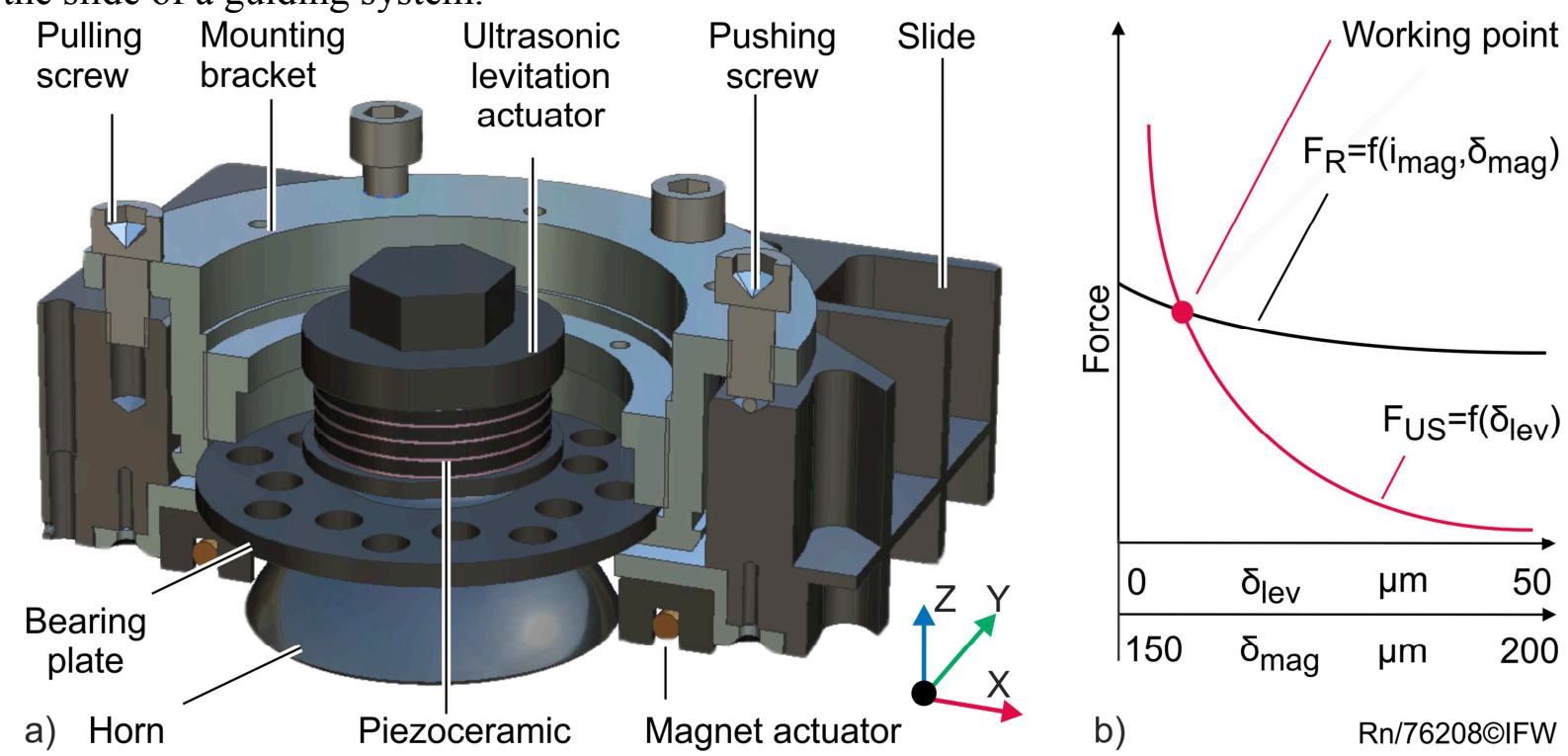

Figure 1: a) USLM actuator, b) Working characteristic

The ultrasonic levitation actuator oscillates in one of its eigenmodes excited by a controlled piezo-ceramic. This results in a contraction/dilation of the whole actuator. A few micrometres vibration amplitude induce the ultrasonic squeeze film levitation effect between the horn and the guiding plane. The ultrasonic actuator is designed to provide up to $6 \mathrm{~N} / \mathrm{cm}^{2}$ levitation force density at $10 \mu \mathrm{m}$ air gap. The diameter of the levitation surface is set to $80 \mathrm{~mm}$, i.e. each actuator theoretically generates $300 \mathrm{~N}$ repellent forces. Resulting from its theoretical minimal working air gap of $10 \mu \mathrm{m}$, the ultrasonic actuators must be oriented relatively to the guiding plane within micrometres and micro-degrees. To comply with this requirements, each actuator is mounted on an adjustable mounting bracket. The mounting bracket is bolted by three pulling- and three pushing screws. Therefore, the actuators are adjustable in the $\mathrm{z}$-direction and the rotational angles around the $\mathrm{x}$ - and $\mathrm{y}$-axes relative to the slide. The rough adjustment is calculated from the measured plane deviation, the geometry of the mounting bracket and the pitch of the fine thread mounting screws. The fine adjustment is conducted by applying additional torque to the pushing screws. This results in additional stresses within the mounting bracket flange, which cause a sensitively adjustable deflection.

The electromagnetic actuator is mounted concentric to the ultrasonic horn. Its force is oriented antagonistic to the ultrasonic levitation force. Fig. 1 (b) shows the working behaviour of the USLM actuator. Both, the levitation force $F_{U S}$ and the magnetic reluctance forces $F_{R}$ act proportionally to the reciprocal of their squared air gap $\delta_{\mathrm{US}}{ }^{2}$ resp. $\delta_{\mathrm{mag}}{ }^{2}$. As shown above, the air gaps $\delta_{\mathrm{US}}$ and $\delta_{\mathrm{mag}}$ are mechanically coupled by the mounting. The offset between them is set to $150 \mu \mathrm{m}$. Because of the antagonistic orientation of the ultrasonic levitation and magnetic forces, a stable working point occurs at the crossing of both characteristic lines. The force level of the electromagnet is controllable by the magnet current $i_{\text {mag. }}$. The resulting magnet force act as an adaptable preload, set to the ultrasonic levitation bearing. Depending on the applied preload the air gap of the USLM actuator is adjustable. The cut-off frequency of the magnetic force generation is set at $4 \mathrm{kHz}$. Thus, the electromagnet can additionally act as a highly dynamic disturbance-force compensation actuator. 


\section{Concept validation: 3 DOF adjustable planar guide}

The combined actuator described above is designed for the use in an active guiding system. Fig. 2 (a) shows the concept of an active and contactless 5 DOF adjustable guiding system.

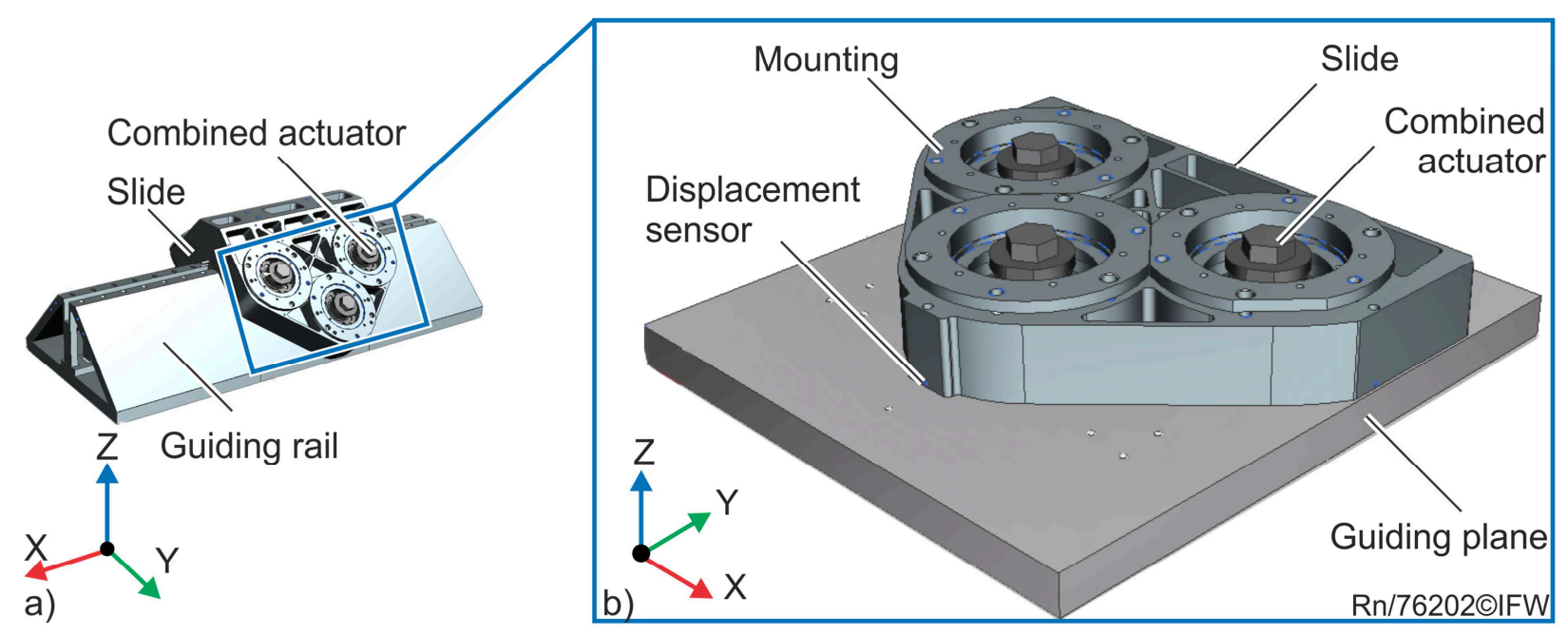

Figure 2: a) 5 DOF adjustable linear guide, b) 3 DOF adjustable planar-guide

Six combined actuators are mounted on a v-shaped slide. Based on the described working behaviour, the adjustable air gaps are used to control the position of the slide in y- and z- direction, in addition to the rotations around all coordinate axes. The free $\mathrm{x}$-direction is served by a linear direct drive, mounted on the notch on the top of the guiding rail. The length of the slide amounts to $400 \mathrm{~mm}$, its travelling distance up to $600 \mathrm{~mm}$. The possible load is restricted by the capabilities of the ultrasonic levitation actuators. Additional to the estimated mass of the slide $(87 \mathrm{~kg})$, a carrying load of up to $40 \mathrm{~kg}$ can be estimated from the calculated ultrasonic levitation actuator force generation. For validation of the concept, a simplified prototype is developed. The simplification is conducted by realising one flank of the v-shaped linear guide (Fig. 2 (a)) as an adjustable 3 DOF fine positioning planar guide (Fig. 2 (b)). Three USLM actuators serve to position the slide in zdirection as well as the rotation around the $\mathrm{x}$ - and $\mathrm{y}$-axis. All dimensioning of the actuators and the design of the planar slide are assumed from the linear guide design. By this, it can be estimated, that the USLM actuators act as they does in the 5 DOF adjustable guiding design. Only the modified mass distribution causes a differing preload of the actuators. To achieve a directly comparable behaviour this effect can be compensated by an additional magnetic preload. In contrast to the adjustable planar guide, the actuation of the linear guide is over-determined. This requires adaptions of the control system architecture. The v-shaped design of the 5 DOF slide cause additional mode shapes which are not represented by the simplified model. Further research will investigate an active damping of these mode shapes. These capabilities are not applicable to the simplified system.

\section{Prototypical implementation and experimental validation}

To validate the USLM active guiding concept, the positioning behaviour of the adjustable planar guide is investigated. In this application the air gaps of the USLM actuators are controlled by three independent PID- controllers. Fig. 3 (a) illustrates the structure of the control system. 

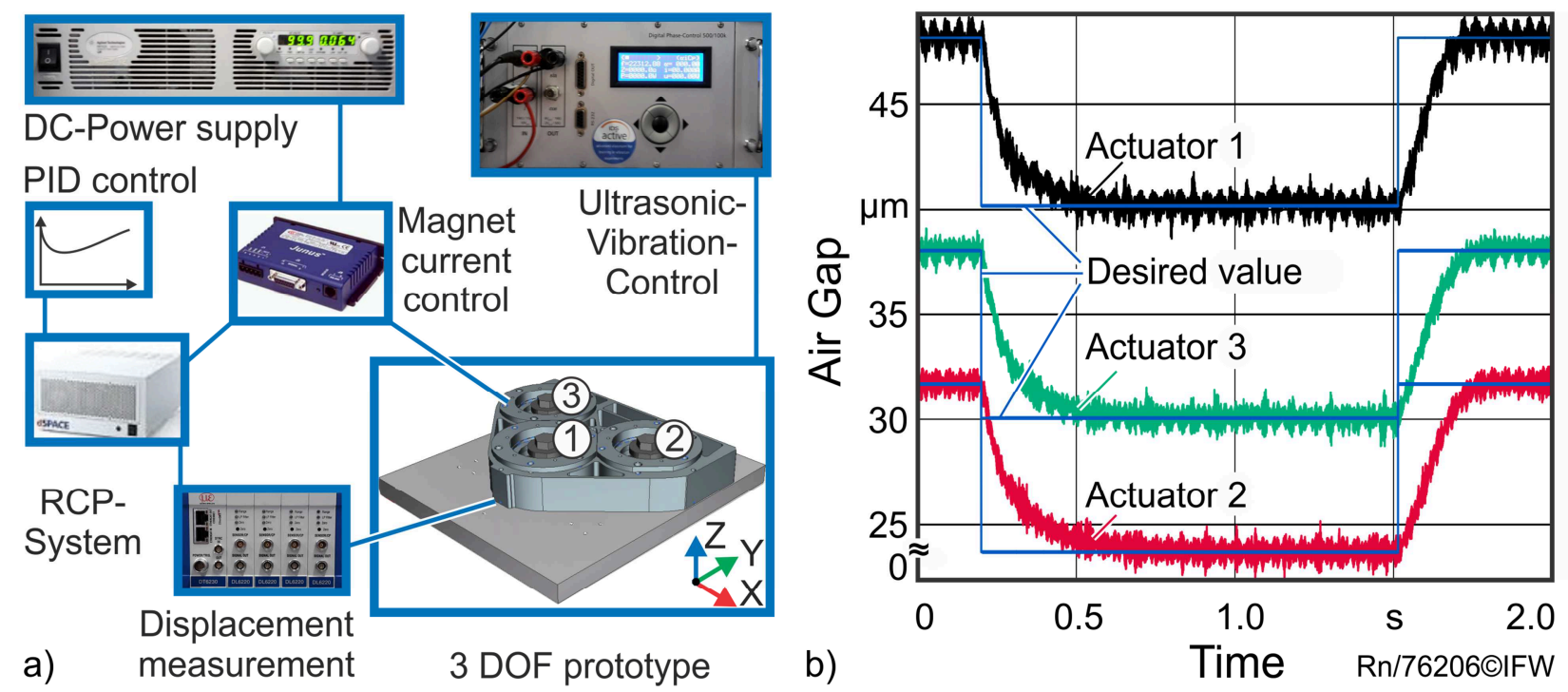

Figure 3: a) Overview of the control system, b) Positioning step response

The ultrasonic actuators are driven by a specific vibration controller, as described in [11]. The controller generates a constant vibration amplitude which results in the described ultrasonic levitation force characteristic (Fig. 1, b). The magnet actuators are driven by conventional four quadrant switching current controllers. Three capacitive displacement sensors measure the distance between the slide and its guiding plane. Each measured deflection corresponds to the air gap of the nearest actuator.

For each pair an own PID-displacement controller is designed. A Rapid Control Prototyping System (RCP System) captures the displacements, calculates the three PID-controller and outputs the new desired values to the magnet current controllers. Fig. 3 (b) shows the step responses for a collective step of $\pm 8 \mu \mathrm{m}$. Before setting the desired value step at $0.2 \mathrm{~s}$ the actual value is set as desired value. Thus, the measured air gaps show the natural deflection of the slide, depending on its mass and the actual ultrasonic forces. Additional magnetic preload is not applied. At $0.2 \mathrm{~s}$ a step of $-8 \mu \mathrm{m}$ is set to the desired position of all controlled deflections. The controllers apply a magnetic preload to move the current working point to the desired position.

The desired position is reached within $0.4 \mathrm{~s}$, the control loop works stationary exact. The step back at $1.5 \mathrm{~s}$ is completed within $0.25 \mathrm{~s}$. The reason for this differing behaviour is to be found in the nonlinear ultrasonic force characteristic (see Fig. 1 (b)). While the air gap is reduced, the error signal of the position controller decreases. Simultaneously the ultrasonic forces are steeply rising. Thus, a reducing airgap increase the time, which is needed to achieve a stationary exact behaviour. A noise amplitude level of $\pm 1 \mu \mathrm{m}$ is superimposed to all deflection signals. It still needs to be analysed if these disturbances are actual deflections caused by the ultrasonic levitation principle itself or are located in the measure- and control environment.

The deflections of the USLM actuators exhibits different levels. The balance point of the slide is located near an imaginary axis through the actuators 2 and 3 (see Fig 3 (a)). Thus, these actuators carry the larger part of the slide's mass. This results in a larger natural preload and lower air gap of these actuators in relation to actuator 1. Inaccurately manufactured and oriented guiding plane and ultrasonic horns cause further differences. The maximum identified ultrasonic force level amounts to $130 \mathrm{~N}$ for each actuator, instead of the theoretically estimated $300 \mathrm{~N}$. Measurements on a coordinate measurement machine determined the form deviation of the ultrasonic levitation horns to $10 \mu \mathrm{m}$. Thus, the identified form deviation act in a similar order of magnitude as the theoretically designed air gap of the ultrasonic actuator. As shown in Fig. 1 (b) the ultrasonic force level depends strongly on the actual air gap. The force loss resulting in the deviation of the air gap can be estimated from the known static working behaviour. If the mean air gap is increased from $10 \mu \mathrm{m}$ to $11 \mu \mathrm{m}$, the force loss would amount to $17.5 \%$. Thus it can be estimated that a more precise manufacturing of both, the ultrasonic horns and the guiding plane, will improve the ultrasonic force generation substantially. 


\section{Compliance frequency response of the 3 DOF guiding system}

The compliance is an essential characteristic of a guiding system. In machine tool manufacturing this behaviour usually is described by compliance or stiffness frequency responses. In the following the frequency compliance behaviour of the planar guiding system is investigated.

A number of mode shapes and eigenfrequencies of the guiding system are known from finite element method (FEM) analysis. In a first step the FEM- simulation is validated by assigning them to the measured eigenfrequencies. During the measurement, the slide rests on the guiding plane without an air cushion, all actuators are deactivated. The slide is excited by an impulse hammer and the deflection is measured by a laser-doppler vibrometer. Both, the excitation and the point of deflection measurement, are set near to the centre of gravity of the slide. Fig. 4 shows the measured and simulated compliance frequency response of the mechanical system.

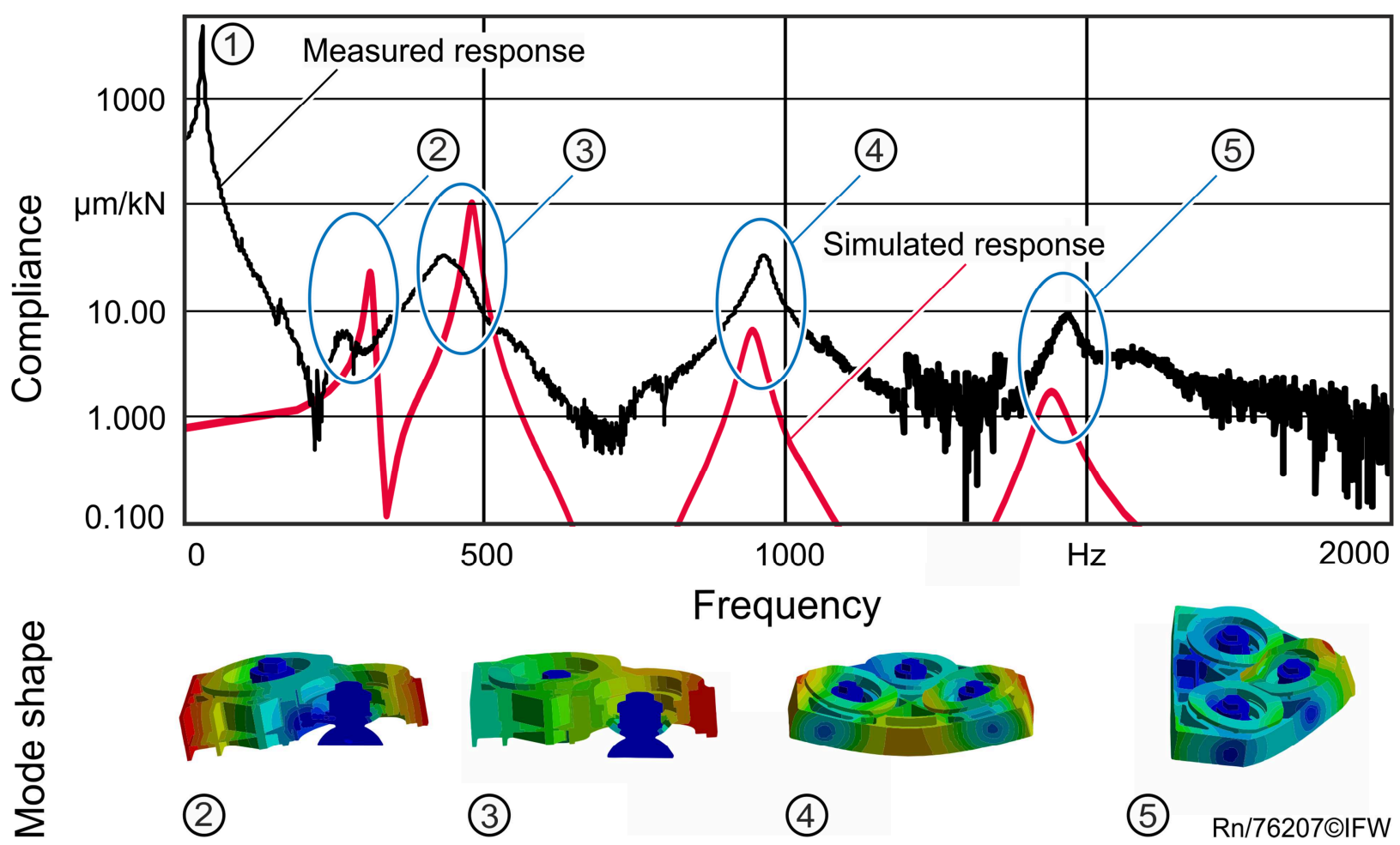

Figure 4: Eigenfrequencies and mode shapes of the mechanical system (deactivated levitation)

In addition to that, important mode shapes of the slide are shown below. Most dominant eigenfrequencies correlate to known mode shapes. Mode (2) represents a mostly rigid body movement of the slide around an x-axis, located near to the centre of gravity (measured $260 \mathrm{~Hz}$, simulated $306 \mathrm{~Hz}$ ). Another mostly rigid body movement occurs in z-direction, normal to the bearing plates of the ultrasonic actuators (meas. $436 \mathrm{~Hz}, \operatorname{sim} .475 \mathrm{~Hz}$ ). A flexure mode shape (4) around the y-axis (meas. $966 \mathrm{~Hz}, \operatorname{sim} .941 \mathrm{~Hz}$ ) and (5) around the x-axis (meas. $1471 \mathrm{~Hz}$, sim. $1438 \mathrm{~Hz}$ ) are assigned, too. The measured frequency response is dominated by an eigenfrequency at $30 \mathrm{~Hz}(1)$. This mode is caused by the test rig, on which the guiding system is mounted. The test rig's structure is not part of the designed simulation model. Thus, this eigenfrequency is not shown in the simulated results. Further differences are caused by a low modelling depth of the FEM-Model and deviations between the modelled and real materials. However, the simulated results show the assigned eigenfrequencies in an adequate accuracy.

Subsequently, the frequency response of the activated guiding is evaluated. The deflection measurement is conducted using the integrated sensors instead of the laser-vibrometer in order to reduce the distortion caused by the eigenmode of the test rig. Fig. 5 shows the response characteristic for an excitation at the centre of gravity to the deflection of sensor 2 (cp. Fig. 3 (a)). 

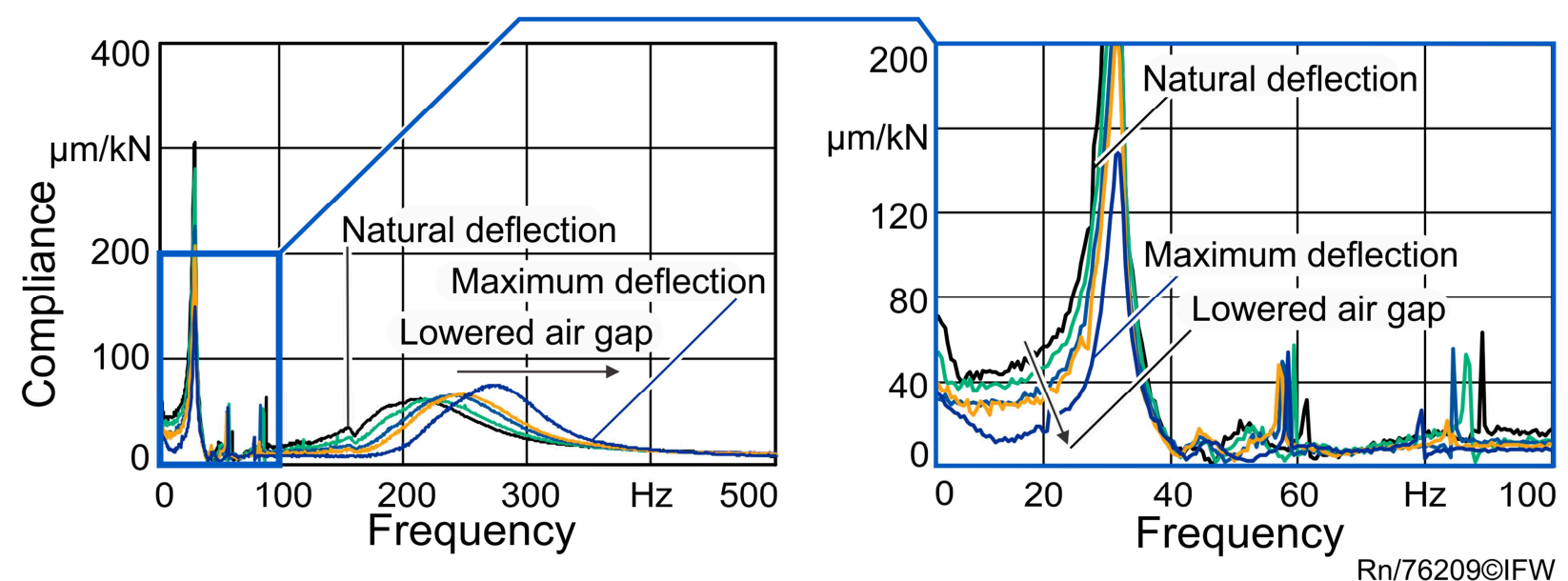

Figure 5: Frequency response of the guiding system (activated levitation)

The response characteristic is measured at varying air gaps. The z-position of the slide is lowered in $2 \mu \mathrm{m}$ steps from $0 \mu \mathrm{m}$ down to $-8 \mu \mathrm{m}$ relative to the natural deflection. The frequency response is dominated by the test rig eigenfrequency at $30 \mathrm{~Hz}$. In the tested setting, the oscillation amplitude of the test rig overstrained the capabilities of the guiding system.

As a result of the varied bearing conditions caused by the activated levitation, the rigid body movement eigenfrequency (cp. Fig. 4, (2)) is replaced by a strongly damped eigenfrequency between $200 \mathrm{~Hz}$ and $280 \mathrm{~Hz}$ (see Fig. 5). Setting a smaller air gap increases its frequency: See Fig. 1 (b)), the force characteristic of the combined actuator is strongly nonlinear. The compliance is determined by the rise of the ultrasonic force characteristic at the actual working point. A lower air gap or higher preload decreases the compliance. A higher preload level reduces the backlash from the test rig eigenmode too. As shown, the maximum compliance is reduced from $300 \mu \mathrm{m} / \mathrm{kN}$ at natural deflection down to $140 \mu \mathrm{m} / \mathrm{kN}$ at maximum deflection.

Excluding the rigid body eigenfrequency and the disturbance from the test rig oscillation, the measured compliance of less than $50 \mu \mathrm{m} / \mathrm{kN}$ for the natural deflection shows a comparable compliance to aerostatic guidings [5]. As estimated from the force characteristic, the compliance is substantially reduced to less than $25 \mu \mathrm{m} / \mathrm{kN}$ by setting an additional preload. The level of preload is restricted by the maximum available ultrasonic forces.

\section{Summary and Conclusion}

The shown prototype exhibits the functionality and dynamic capabilities of novel ultrasonic levitation magnetic actuators. On the basis of the static characteristic of these actuators, the estimated working behaviour of the guiding system is derived and proven by measurement. The control system positions the guiding system slide in 3 DOF by using a PID-Controller. The measured compliance frequency response shows a comparable compliance level to conventional aerostatic guiding systems. The compliance behaviour is further improved by the applied preload. According to the working principle of the USLM actuator, the level of preload is restricted by its maximum ultrasonic forces.

Further potential is identified to improve the compliance behaviour of the guiding system. It can be estimated that a more precise manufacturing and adjustment reduces the possible working air gap. Thus, the available forces of the ultrasonic levitation actuators are increased. By setting a higher level of preload at once, the guiding systems compliance will be improved substantially.

The dynamic capabilities of the magnet actuators are not fully exploited by the PID-controller so far. In further works, a more sophisticated controller design based on a state control is intended to improve the dynamic behaviour of the guiding systems.

However, the practicability of the presented prototype is restricted as three DOF are not actuated yet. Further works transfer the results, extracted from the investigations on the 3 DOF adjustable prototype to the development and realisation of a fully actuated 5 DOF adjustable USLM linear guiding concept. 


\section{Acknowledgement}

The authors thank the German Research Foundation (DFG) for funding this research (DE447/106-1, WA 564/27-1).

\section{References}

[1] O. Riemer, Advances in ultraprecision manufacturing, Procedings Japan Society for Precision Engineering (2011).

[2] F. Wardle, Ultra precision bearings, Cambridge, Woodhead Publishing, 2015.

[3] D. Dornfeld, D.-E. Lee, Precision manufacturing, New York, Springer, 2008.

[4] M. Weck, Werkzeugmaschinen - Konstruktion und Berechnung, Berlin, Springer, 2002.

[5] S.-K. Ro, S. Kim, Y. Kwak, C. H. Park, A linear air bearing stage with active magnetic preloads for ultraprecise straight motion, CIRP-CAT 200734 (2010), no. 1, p.186-194.

[6] Y. Altintas, A. Verl, C. Brecher, L. Uriarte, G. Pritschow, Machine tool feed drives, CIRP Annals - Manufacturing Technology 60 (2011), no. 2, p.779-796.

[7] F. Hackelöer, Erweiterung der Einsatzmöglichkeiten von Magnetführungen, Garbsen, PZHVerlag, 2014.

[8] B. Denkena, D. Dahlmann, R. Krueger, Electromagnetic Levitation Guide for Use in UltraPrecision Milling Centres, Procedia CIRP 37 (2015), p.199-204.

[9] S. Mojrzisch, F. Hackelöer, H.-C. Möhring, B. Denkena, J. Wallaschek, Führung, Patent DE102011015973B4, 2013.

[10] S. Mojrzisch, I. Ille, J. Wallaschek, B. Denkena, A hybrid ultrasonic squeeze film and magnetic levitation actuator for machine guideways, 2013 IEEE International Ultrasonics Symposium (IUS), 2013, p.485-487.

[11]I. Ille, J. Twiefel, Model-based Feedback Control of an Ultrasonic Transducer for Ultrasonic Assisted Turning Using a Novel Digital Controller, Proceedings of the 2015 ICU International Congress on Ultrasonics, Metz, France (2015), p.63-67. 